CARPATHIAN J. MATH.

Volume 37 (2021), No. 3,

Pages 449 - 461

\title{
A fast viscosity forward-backward algorithm for convex minimization problems with an application in image recovery
}

\author{
PACHARA JAILOKA, SUtheP SuANTAi and AdisAK HANJing
}

\begin{abstract}
The purpose of this paper is to invent an accelerated algorithm for the convex minimization problem which can be applied to the image restoration problem. Theoretically, we first introduce an algorithm based on viscosity approximation method with the inertial technique for finding a common fixed point of a countable family of nonexpansive operators. Under some suitable assumptions, a strong convergence theorem of the proposed algorithm is established. Subsequently, we utilize our proposed algorithm to solving a convex minimization problem of the sum of two convex functions. As an application, we apply and analyze our algorithm to image restoration problems. Moreover, we compare convergence behavior and efficiency of our algorithm with other well-known methods such as the forward-backward splitting algorithm and the fast iterative shrinkage-thresholding algorithm. By using image quality metrics, numerical experiments show that our algorithm has a higher efficiency than the mentioned algorithms.
\end{abstract}

\section{INTRODUCTION}

Over the past decades, many optimization algorithms have been efficiently developed for solving inverse problems in signal and image processing, see $[4,7,8,9,10,11,12]$, for instance. Many problems in image processing, especially the image restoration problem, can be formulated as the following model:

$$
y=B x+\varepsilon,
$$

where $x \in \mathbb{R}^{N}$ is an original image, $y \in \mathbb{R}^{M}$ is the observed image, $\varepsilon$ is an additive noise and $B \in \mathbb{R}^{M \times N}$ is the blurring operation. To approximate the original image in (1.1), we need to minimize the value of $\varepsilon$ by using the LASSO technique [22]:

$$
\min _{x \in \mathbb{R}^{N}}\left\{\frac{1}{2}\|y-B x\|_{2}^{2}+\lambda\|x\|_{1}\right\},
$$

where $\lambda$ is a positive parameter, $\|\cdot\|_{1}$ is the $l_{1}$-norm and $\|\cdot\|_{2}$ is the Euclidean norm. In general case, (1.2) can be formally considered as the following unconstrained convex minimization problems.

Problem 1.1. Let $f_{1}: \mathbb{R}^{N} \rightarrow \mathbb{R}$ be a convex and differentiable function with a $L$-Lipschitz continuous gradient $\nabla f_{1}$ and let $f_{2}: \mathbb{R}^{N} \rightarrow \mathbb{R} \cup\{\infty\}$ be a proper lower semi-continuous and convex function. The problem is modeled as follows:

$$
\min _{x \in \mathbb{R}^{N}}\left\{f_{1}(x)+f_{2}(x)\right\}
$$

Received: 18.08.2020. In revised form: 14.11.2020. Accepted: 21.11.2020

2010 Mathematics Subject Classification. 47J25, 65K05, 47H10.

Key words and phrases. convex minimization problems, fixed points, image restoration problems, forward-backward algorithms, viscosity approximation, strong convergence.

Corresponding author: Adisak Hanjing; adisak_h@cmu.ac.th 
It can be shown [7] that if $f_{1}(x)+f_{2}(x) \rightarrow \infty$ as $\|x\| \rightarrow \infty$, then Problem 1.1 has at least one solution. In addition, a solution $x^{*}$ of Problem 1.1 is characterized by the fixed point equation:

$$
x^{*}=\operatorname{prox}_{c f_{2}}\left(I-c \nabla f_{1}\right)\left(x^{*}\right),
$$

where $c>0, \operatorname{prox}_{f_{2}}$ is the proximity operator of $f_{2}$ and $I$ stands for the identity operator on $\mathbb{R}^{N}$. To solve Problem 1.1, the above equation leads to the following iteration:

$$
x_{n+1}=\underbrace{\operatorname{prox}_{c_{n} f_{2}}}_{\text {backward step }} \underbrace{\left(I-c_{n} \nabla f_{1}\right)\left(x_{n}\right)}_{\text {forward step }}, \quad n \geq 1,
$$

where $x_{1} \in \mathbb{R}^{N}$ and $0<c_{n}<2 / L$. This method is known as the forward-backward splitting algorithm (FBSA) [13]. In literature, the FBSA is also called an iterative denoising method [10], a Landweber iteration [9] or a fixed point continuation algorithm [12].

Beck and Teboulle [4] introduced the fast iterative shrinkage-thresholding algorithm (FISTA) for solving Problem 1.1 by using an inertial technique as follows:

$$
\left\{\begin{array}{l}
y_{n}=\operatorname{prox}_{\frac{1}{L} f_{2}}\left(I-\frac{1}{L} \nabla f_{1}\right)\left(x_{n}\right), \\
t_{n+1}=\frac{1+\sqrt{1+4 t_{n}^{2}}}{2}, \quad \theta_{n}=\frac{t_{n}-1}{t_{n+1}}, \quad n \geq 1, \\
x_{n+1}=y_{n}+\theta_{n}\left(y_{n}-y_{n-1}\right), \quad n \geq 1
\end{array}\right.
$$

where $x_{1}=y_{0} \in \mathbb{R}^{N}, t_{1}=1$. They proved the rate of convergence of the FISTA and applied the FISTA to image restoration problems. After that, Liang and Schonlieb [14] modified the FISTA by replacing $t_{n+1}=\left(p+\sqrt{q+r t_{n}^{2}}\right) / 2$ where $p, q>0$ and $0<r \leq 4$, and proved a weak convergence result.

It is worth noting from the above review that the convex minimization problem is related to the fixed point problem. Also, we know that a forward-backward operator $T:=\operatorname{prox}_{c f_{2}}\left(I-c \nabla f_{1}\right)$ is nonexpansive if $0<c<2 / L$. So the study on fixed point problems for the class of nonexpansive operators plays an important role in creating optimization methods. In the setting of a Hilbert space $\mathcal{H}$, let $T: \mathcal{H} \rightarrow \mathcal{H}$ be a nonexpansive operator. The Mann iterative method is a well-known procedure, in order to approximate fixed points of $T$; however, Mann iteration guarantees only weak convergence for the class of nonexpansive operators. To get strong convergence, many authors often used the so-called viscosity approximation method $[16,26]$ as follows:

$$
x_{n+1}=\gamma_{n} g\left(x_{n}\right)+\left(1-\gamma_{n}\right) T x_{n}, \quad n \geq 1,
$$

where $x_{1} \in \mathcal{H}$ and $g: \mathcal{H} \rightarrow \mathcal{H}$ is a contraction and $\left\{\gamma_{n}\right\}$ is a suitable sequence in $(0,1)$. In particular, if $T:=\operatorname{prox}_{c f_{2}}\left(I-c \nabla f_{1}\right)$, then we call (1.7) the viscosity forward-backward algorithm (VFBA).

In this paper, inspired and motivated by these researches, we aim to invent a new accelerated algorithm for the convex minimization problem which can be applied to the image recovery problem. The paper is organized as follows. In Section 2, basic definitions, notations, and some useful tools for proving our main results are given. In Section 3, we introduce a viscosity-type algorithm with the inertial technical term for finding a common fixed point of a countable family of nonexpansive operators in a Hilbert space. Furthermore, we prove a strong convergence result of the proposed algorithm under the condition $(Z)$ and some control conditions. Subsequently, we apply our proposed algorithm to solving an unconstrained minimization problem of the sum of two convex functions. Finally, in Section 4, we analyze and illustrate the performance of our algorithm (FVFBA) for image restoration problems, and also compare its efficiency with the FBSA, the VFBA 
and the FISTA. Numerical experiments show that our algorithm has a higher efficiency than above-mentioned algorithms by using PSNR and SSIM image quality metrics.

\section{PRELIMINARIES}

Throughout this paper, we denote by $\mathbb{R}$ and $\mathbb{N}$ the set of real numbers and the set of positive integers, respectively. I stands for the identity operator on a Hilbert space. We assume that $\mathcal{H}$ is a real Hilbert space with an inner product $\langle\cdot, \cdot\rangle$ and the induced norm $\|\cdot\|$. Denote weak and strong convergence of a sequence $\left\{x_{n}\right\} \subset \mathcal{H}$ to $x \in \mathcal{H}$ by $x_{n} \rightarrow x$ and $x_{n} \rightarrow x$, respectively. If $f: \mathcal{H} \rightarrow \mathbb{R}$ is a differentiable function, then we denote the gradient of $f$ by $\nabla f$.

The following classes of nonlinear operators are needed in this work.

Definition 2.1. An operator $T: \mathcal{H} \rightarrow \mathcal{H}$ is said to be

(i) Lipschitz continuous if there exists $L>0$ such that

$$
\|T x-T y\| \leq L\|x-y\|, \forall x, y \in \mathcal{H}
$$

(ii) a contraction if $T$ is $L$-Lipschitz continuous where $L<1$,

(iii) nonexpansive if $T$ is 1-Lipschitz continuous.

Let $T: \mathcal{H} \rightarrow \mathcal{H}$ be an operator. A fixed point of $T$ is a point in $\mathcal{H}$ which is mapped to itself by $T$, and the set of all fixed points of $T$ is denoted by

$$
\operatorname{Fix}(T):=\{x \in \mathcal{H}: x=T x\} .
$$

The operator $I-T$ is called demiclosed at zero if for any sequence $\left\{x_{n}\right\}$ in $\mathcal{H}$ which converges weakly to $x$, and if the sequence $\left\{x_{n}-T x_{n}\right\}$ converges strongly to 0 , then $x \in \operatorname{Fix}(T)$. It is known [19] that if $T$ is a nonexpansive operator, then $I-T$ is demiclosed at zero. Let $\left\{T_{n}: \mathcal{H} \rightarrow \mathcal{H}\right\}$ be such that $\emptyset \neq \operatorname{Fix}(T) \subset \bigcap_{n=1}^{\infty} \operatorname{Fix}\left(T_{n}\right)$. Then, $\left\{T_{n}\right\}$ is said to satisfy the NST-condition (I) with $T$ [18] if for each bounded sequence $\left\{x_{n}\right\} \subset \mathcal{H}$,

$$
\lim _{n \rightarrow \infty}\left\|x_{n}-T_{n} x_{n}\right\|=0 \text { implies } \lim _{n \rightarrow \infty}\left\|x_{n}-T x_{n}\right\|=0 .
$$

Now, we give an important condition for proving our main result.

Definition 2.2. [1,2] A sequence $\left\{T_{n}: \mathcal{H} \rightarrow \mathcal{H}\right\}$ with a nonempty common fixed point set is said to satisfy the condition (Z) if whenever $\left\{x_{n}\right\}$ is a bounded sequence in $\mathcal{H}$ such that

$$
\lim _{n \rightarrow \infty}\left\|x_{n}-T_{n} x_{n}\right\|=0
$$

it follows that every weak cluster point of $\left\{x_{n}\right\}$ belongs to $\bigcap_{n=1}^{\infty} \operatorname{Fix}\left(T_{n}\right)$.

Let $K$ be a nonempty closed convex subset of $\mathcal{H}$. The metric projection $P_{K}$ from $\mathcal{H}$ onto $K$, is defined for each $x \in \mathcal{H}, P_{K} x$ is the unique element in $K$ such that

$$
\left\|x-P_{K} x\right\|=\inf \{\|x-y\|: y \in K\} .
$$

It is known that $u=P_{K} x$ if and only if $\langle x-u, y-u\rangle \leq 0$ for all $y \in K$.

Let us recall the definition of the proximity operator and its properties.

Definition $2.3([15,3])$. Let $f: \mathcal{H} \rightarrow \mathbb{R} \cup\{\infty\}$ be a proper lower semi-continuous and convex function. The proximity operator of $f$, denoted by $\operatorname{prox}_{f}$ is defined for each $x \in \mathcal{H}$, $\operatorname{prox}_{f} x$ is the unique optimal solution of the minimization problem

$$
\underset{y \in \mathcal{H}}{\operatorname{minimize}} f(y)+\frac{1}{2}\|x-y\|^{2} .
$$


The proximity operator can be formulated in the equivalent form:

$$
\operatorname{prox}_{f}=(I+\partial f)^{-1}
$$

where $\partial f$ is the subdifferential of $f$ defined by

$$
\partial f(x):=\{u \in \mathcal{H}: f(x)+\langle u, y-x\rangle \leq f(y), \forall y \in \mathcal{H}\},
$$

for all $x \in \mathcal{H}$. Let $c>0$. We also know that $\operatorname{prox}_{c f}$ is (firmly) nonexpansive and

$$
\operatorname{Fix}\left(\operatorname{prox}_{c f}\right)=\operatorname{Argmin} f:=\{x \in \mathcal{H}: f(x) \leq f(y), \forall y \in \mathcal{H}\} .
$$

Let $K \subset \mathcal{H}$ be a nonempty closed convex set. In particular, if $f:=i_{K}$ is an indicator function on $K$ (defined by $i_{K}(x)=0$ if $x \in K$; otherwise $i_{K}(x)=\infty$ ), then $\operatorname{prox}_{c f}=P_{K}$.

We end this section by providing useful lemmas for proving our convergence results.

Lemma 2.1 ([5]). Let $f_{1}: \mathcal{H} \rightarrow \mathbb{R}$ be a convex and differentiable function with a $L$-Lipschitz continuous gradient $\nabla f_{1}$ and let $f_{2}: \mathcal{H} \rightarrow \mathbb{R} \cup\{\infty\}$ be a proper lower semi-continuous and convex function. Let $T_{n}:=\operatorname{prox}_{c_{n} f_{2}}\left(I-c_{n} \nabla f_{1}\right)$ and $T:=\operatorname{prox}_{c f_{2}}\left(I-c \nabla f_{1}\right)$, where $c_{n}, c \in(0,2 / L)$ with $c_{n} \rightarrow c$ as $n \rightarrow \infty$. Then $\left\{T_{n}\right\}$ satisfies the NST-condition (I) with $T$.

Lemma 2.2 ([23]). Let $x, y \in \mathcal{H}$ and $t \in[0,1]$. Then the following properties hold on $\mathcal{H}$ :

(i) $\|t x+(1-t) y\|^{2}=t\|x\|^{2}+(1-t)\|y\|^{2}-t(1-t)\|x-y\|^{2}$;

(ii) $\|x \pm y\|^{2}=\|x\|^{2} \pm 2\langle x, y\rangle+\|y\|^{2}$;

(iii) $\|x+y\|^{2} \leq\|x\|^{2}+2\langle y, x+y\rangle$.

Lemma 2.3 ([21]). Let $\left\{a_{n}\right\}$ be a sequence of nonnegative real numbers and $\left\{b_{n}\right\}$ a sequence of real numbers. Let $\left\{t_{n}\right\}$ be a sequence of real numbers in $(0,1)$ such that $\sum_{n=1}^{\infty} t_{n}=\infty$. Assume that

$$
a_{n+1} \leq\left(1-t_{n}\right) a_{n}+t_{n} b_{n}, \quad n \in \mathbb{N} .
$$

If $\lim \sup _{i \rightarrow \infty} b_{n_{i}} \leq 0$ for every subsequence $\left\{a_{n_{i}}\right\}$ of $\left\{a_{n}\right\}$ satisfying

$$
\liminf _{i \rightarrow \infty}\left(a_{n_{i}+1}-a_{n_{i}}\right) \geq 0
$$

then $\lim _{n \rightarrow \infty} a_{n}=0$.

\section{MethodS AND CONVERGENCE RESULTS}

In this section, based on the viscosity approximation method we introduce a new accelerated algorithm using the inertial technique for finding a common fixed point of a countable family of nonexpansive operators satisfying the condition (Z) in a Hilbert space. A strong convergence theorem is proved under some suitable control conditions. After that, we apply the proposed algorithm to solving a convex minimization problem of the sum of two convex functions.

We begin by setting the following hypotheses:

- $\mathcal{H}$ is a real Hilbert space;

- $\left\{T_{n}: \mathcal{H} \rightarrow \mathcal{H}\right\}$ is a family of nonexpansive operators;

- $\Gamma:=\bigcap_{n=1}^{\infty} \operatorname{Fix}\left(T_{n}\right) \neq \emptyset$;

- $\left\{T_{n}\right\}$ satisfies the condition $(Z)$;

- $g: \mathcal{H} \rightarrow \mathcal{H}$ is a $k$-contraction, where $0<k<1$.

In optimization theory, to speed up the convergence of iterative methods, many mathematicians often use the inertial-type extrapolation [20,17] by supplementing the technical term $\theta_{n}\left(x_{n}-x_{n-1}\right)$. The control parameter $\theta_{n}$ is called an inertial parameter, which controls the momentum $x_{n}-x_{n-1}$. Now, we are ready to introduce a modified inertial viscosity approximation method as follows: 


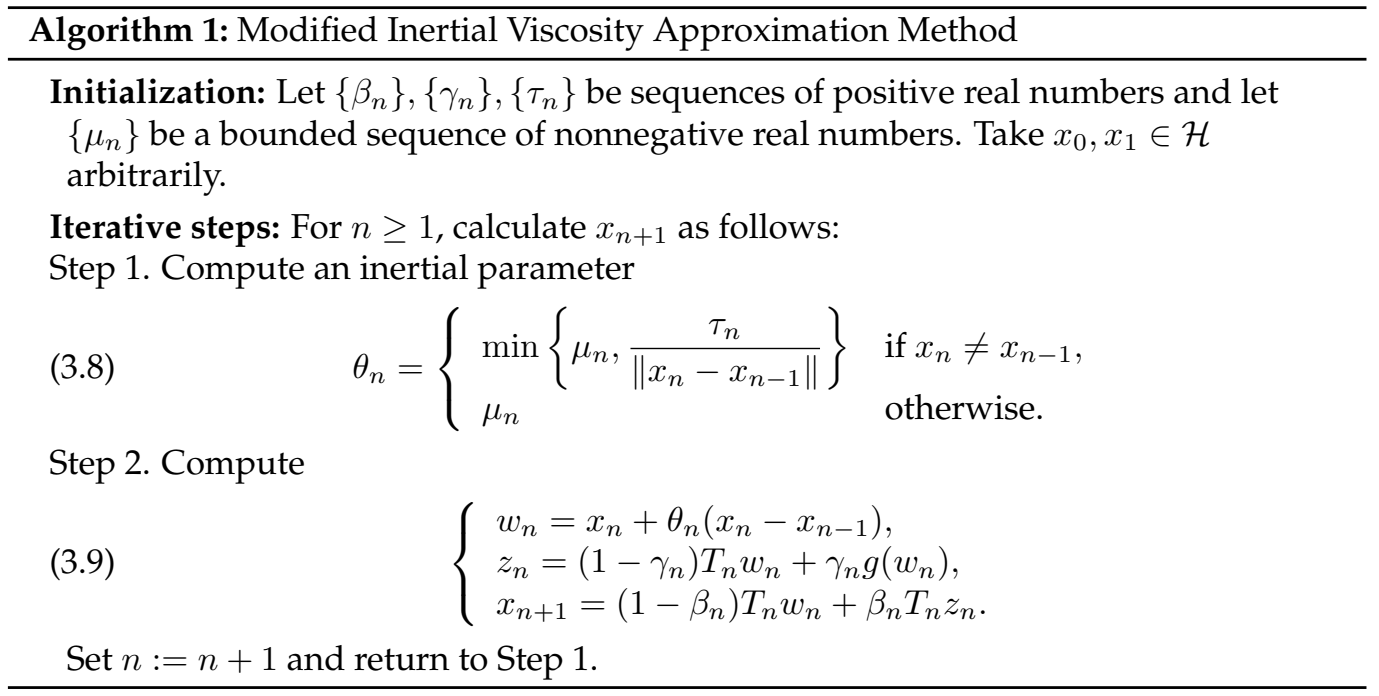

A strong convergence result is established by the following theorem.

Theorem 3.1. Any sequence $\left\{x_{n}\right\}$ generated by Algorithm 1 converges strongly to an element $x^{*} \in \Gamma$, where $x^{*}=P_{\Gamma} g\left(x^{*}\right)$, provided that the sequences $\left\{\beta_{n}\right\},\left\{\gamma_{n}\right\}$ and $\left\{\tau_{n}\right\}$ satisfy the following conditions:

(C1) $0<\epsilon_{1} \leq \beta_{n} \leq \epsilon_{2}<1$;

(C2) $0<\gamma_{n}<1, \lim _{n \rightarrow \infty} \beta_{n} \gamma_{n}=0$ and $\sum_{n=1}^{\infty} \beta_{n} \gamma_{n}=\infty$;

(C3) $\lim _{n \rightarrow \infty} \frac{\tau_{n}}{\beta_{n} \gamma_{n}}=0$.

Proof. One can see that the operator $P_{\Gamma} g$ is a contraction. By the Banach contraction principle, there is a unique point $x^{*} \in \Gamma$ such that $x^{*}=P_{\Gamma} g\left(x^{*}\right)$. Thus, $x^{*}=T_{n} x^{*}$ for all $n$. Firstly, we show that $\left\{x_{n}\right\}$ is bounded. From (3.9), we have

$$
\begin{aligned}
\left\|z_{n}-x^{*}\right\| & \leq \gamma_{n}\left\|g\left(w_{n}\right)-x^{*}\right\|+\left(1-\gamma_{n}\right)\left\|T_{n} w_{n}-x^{*}\right\| \\
& \leq \gamma_{n}\left\|g\left(w_{n}\right)-g\left(x^{*}\right)\right\|+\gamma_{n}\left\|g\left(x^{*}\right)-x^{*}\right\|+\left(1-\gamma_{n}\right)\left\|T_{n} w_{n}-x^{*}\right\| \\
& \leq \gamma_{n} k\left\|w_{n}-x^{*}\right\|+\gamma_{n}\left\|g\left(x^{*}\right)-x^{*}\right\|+\left(1-\gamma_{n}\right)\left\|w_{n}-x^{*}\right\| \\
& =\left(1-\gamma_{n}(1-k)\right)\left\|w_{n}-x^{*}\right\|+\gamma_{n}\left\|g\left(x^{*}\right)-x^{*}\right\|,
\end{aligned}
$$

which implies that

$$
\begin{aligned}
\left\|x_{n+1}-x^{*}\right\| & \leq\left(1-\beta_{n}\right)\left\|T_{n} w_{n}-x^{*}\right\|+\beta_{n}\left\|T_{n} z_{n}-x^{*}\right\| \\
& \leq\left(1-\beta_{n}\right)\left\|w_{n}-x^{*}\right\|+\beta_{n}\left\|z_{n}-x^{*}\right\| \\
& \leq\left(1-\beta_{n} \gamma_{n}(1-k)\right)\left\|w_{n}-x^{*}\right\|+\beta_{n} \gamma_{n}\left\|g\left(x^{*}\right)-x^{*}\right\| \\
& \leq\left(1-\beta_{n} \gamma_{n}(1-k)\right)\left(\left\|x_{n}-x^{*}\right\|+\theta_{n}\left\|x_{n}-x_{n-1}\right\|\right)+\beta_{n} \gamma_{n}\left\|g\left(x^{*}\right)-x^{*}\right\| \\
& \leq\left(1-\beta_{n} \gamma_{n}(1-k)\right)\left\|x_{n}-x^{*}\right\|+\beta_{n} \gamma_{n}\left(\frac{\theta_{n}}{\beta_{n} \gamma_{n}}\left\|x_{n}-x_{n-1}\right\|+\left\|g\left(x^{*}\right)-x^{*}\right\|\right) .
\end{aligned}
$$


By (3.8) and (C3), we have $\frac{\theta_{n}}{\beta_{n} \gamma_{n}}\left\|x_{n}-x_{n-1}\right\| \rightarrow 0$ as $n \rightarrow \infty$ and so there exists $M>0$ such that $\frac{\theta_{n}}{\beta_{n} \gamma_{n}}\left\|x_{n}-x_{n-1}\right\| \leq M$ for all $n \geq 1$. Thus,

$$
\begin{aligned}
\left\|x_{n+1}-x^{*}\right\| & \leq\left(1-\beta_{n} \gamma_{n}(1-k)\right)\left\|x_{n}-x^{*}\right\|+\beta_{n} \gamma_{n}(1-k) \frac{M+\left\|g\left(x^{*}\right)-x^{*}\right\|}{1-k} \\
& \leq \max \left\{\left\|x_{n}-x^{*}\right\|, \frac{M+\left\|g\left(x^{*}\right)-x^{*}\right\|}{1-k}\right\} .
\end{aligned}
$$

By mathematical induction, we deduce that

$$
\left\|x_{n}-x^{*}\right\| \leq \max \left\{\left\|x_{1}-x^{*}\right\|, \frac{M+\left\|g\left(x^{*}\right)-x^{*}\right\|}{1-k}\right\}, \quad \forall n \geq 1 .
$$

This means that $\left\{x_{n}\right\}$ is bounded and hence $\left\{g\left(w_{n}\right)\right\},\left\{T_{n} w_{n}\right\}$ and $\left\{z_{n}\right\}$ are also bounded.

By Lemma 2.2, we have

$$
\begin{aligned}
\left\|z_{n}-x^{*}\right\|^{2} & =\left\|\left(1-\gamma_{n}\right)\left(T_{n} w_{n}-x^{*}\right)+\gamma_{n}\left(g\left(w_{n}\right)-g\left(x^{*}\right)\right)+\gamma_{n}\left(g\left(x^{*}\right)-x^{*}\right)\right\|^{2} \\
& \leq\left\|\left(1-\gamma_{n}\right)\left(T_{n} w_{n}-x^{*}\right)+\gamma_{n}\left(g\left(w_{n}\right)-g\left(x^{*}\right)\right)\right\|^{2}+2 \gamma_{n}\left\langle g\left(x^{*}\right)-x^{*}, z_{n}-x^{*}\right\rangle \\
& \leq\left(1-\gamma_{n}\right)\left\|T_{n} w_{n}-x^{*}\right\|^{2}+\gamma_{n}\left\|g\left(w_{n}\right)-g\left(x^{*}\right)\right\|^{2}+2 \gamma_{n}\left\langle g\left(x^{*}\right)-x^{*}, z_{n}-x^{*}\right\rangle \\
& \leq\left(1-\gamma_{n}(1-k)\right)\left\|w_{n}-x^{*}\right\|^{2}+2 \gamma_{n}\left\langle g\left(x^{*}\right)-x^{*}, z_{n}-x^{*}\right\rangle
\end{aligned}
$$

and

$$
\begin{aligned}
\left\|w_{n}-x^{*}\right\|^{2} & =\left\|x_{n}-x^{*}\right\|^{2}+\theta_{n}^{2}\left\|x_{n}-x_{n-1}\right\|^{2}+2 \theta_{n}\left\langle x_{n}-x^{*}, x_{n}-x_{n-1}\right\rangle \\
& \leq\left\|x_{n}-x^{*}\right\|^{2}+\theta_{n}^{2}\left\|x_{n}-x_{n-1}\right\|^{2}+2 \theta_{n}\left\|x_{n}-x^{*}\right\|\left\|x_{n}-x_{n-1}\right\| .
\end{aligned}
$$

By Lemma 2.2(i) together with (3.10) and (3.11), we have

$$
\begin{aligned}
\left\|x_{n+1}-x^{*}\right\|^{2}= & \left(1-\beta_{n}\right)\left\|T_{n} w_{n}-x^{*}\right\|^{2}+\beta_{n}\left\|T_{n} z_{n}-x^{*}\right\|^{2}-\beta_{n}\left(1-\beta_{n}\right)\left\|T_{n} w_{n}-T_{n} z_{n}\right\|^{2} \\
\leq & \left(1-\beta_{n}\right)\left\|w_{n}-x^{*}\right\|^{2}+\beta_{n}\left\|z_{n}-x^{*}\right\|^{2}-\beta_{n}\left(1-\beta_{n}\right)\left\|T_{n} w_{n}-T_{n} z_{n}\right\|^{2} \\
\leq & \left(1-\beta_{n} \gamma_{n}(1-k)\right)\left\|w_{n}-x^{*}\right\|^{2}+2 \beta_{n} \gamma_{n}\left\langle g\left(x^{*}\right)-x^{*}, z_{n}-x^{*}\right\rangle \\
& -\beta_{n}\left(1-\beta_{n}\right)\left\|T_{n} w_{n}-T_{n} z_{n}\right\|^{2} \\
\leq & \left(1-\beta_{n} \gamma_{n}(1-k)\right)\left\|x_{n}-x^{*}\right\|^{2}+\theta_{n}^{2}\left\|x_{n}-x_{n-1}\right\|^{2} \\
& +2 \theta_{n}\left\|x_{n}-x^{*}\right\|\left\|x_{n}-x_{n-1}\right\|+2 \beta_{n} \gamma_{n}\left\langle g\left(x^{*}\right)-x^{*}, z_{n}-x^{*}\right\rangle \\
& -\beta_{n}\left(1-\beta_{n}\right)\left\|T_{n} w_{n}-T_{n} z_{n}\right\|^{2} \\
= & \left(1-\beta_{n} \gamma_{n}(1-k)\right)\left\|x_{n}-x^{*}\right\|^{2}-\beta_{n}\left(1-\beta_{n}\right)\left\|T_{n} w_{n}-T_{n} z_{n}\right\|^{2} \\
& +\beta_{n} \gamma_{n}(1-k) b_{n},
\end{aligned}
$$

where

$$
\begin{aligned}
b_{n}=\frac{1}{1-k} & \left\{2\left\langle g\left(x^{*}\right)-x^{*}, z_{n}-x^{*}\right\rangle+\left(\frac{\theta_{n}}{\beta_{n} \gamma_{n}}\left\|x_{n}-x_{n-1}\right\|\right) \theta_{n}\left\|x_{n}-x_{n-1}\right\|\right. \\
+ & \left.2\left\|x_{n}-x^{*}\right\|\left(\frac{\theta_{n}}{\beta_{n} \gamma_{n}}\left\|x_{n}-x_{n-1}\right\|\right)\right\} .
\end{aligned}
$$

It follows that

$$
\beta_{n}\left(1-\beta_{n}\right)\left\|T_{n} w_{n}-T_{n} z_{n}\right\|^{2} \leq\left\|x_{n}-x^{*}\right\|^{2}-\left\|x_{n+1}-x^{*}\right\|^{2}+\beta_{n} \gamma_{n}(1-k) M^{\prime},
$$

where $M^{\prime}=\sup \left\{b_{n}: n \in \mathbb{N}\right\}$. 
Let us show that $\left\{x_{n}\right\}$ converges strongly to $x^{*}$. To apply Lemma 2.3, we let $a_{n}:=$ $\left\|x_{n}-x^{*}\right\|^{2}$ and $t_{n}:=\beta_{n} \gamma_{n}(1-k)$. From (3.12), we have the following inequality:

$$
a_{n+1} \leq\left(1-t_{n}\right) a_{n}+t_{n} b_{n} .
$$

Suppose that $\left\{a_{n_{i}}\right\}$ is a subsequence of $\left\{a_{n}\right\}$ such that $\liminf _{i \rightarrow \infty}\left(a_{n_{i}+1}-a_{n_{i}}\right) \geq 0$. Then, by (3.13) and (C2) we have

$$
\begin{aligned}
\limsup _{i \rightarrow \infty} \beta_{n_{i}}\left(1-\beta_{n_{i}}\right)\left\|T_{n_{i}} w_{n_{i}}-T_{n_{i}} z_{n_{i}}\right\|^{2} & \leq \limsup _{i \rightarrow \infty}\left(a_{n_{i}}-a_{n_{i}+1}+\beta_{n_{i}} \gamma_{n_{i}}(1-k) M^{\prime}\right) \\
& \leq \limsup _{i \rightarrow \infty}\left(a_{n_{i}}-a_{n_{i}+1}\right)+(1-k) M^{\prime} \lim _{i \rightarrow \infty} \beta_{n_{i}} \gamma_{n_{i}} \\
& =-\liminf _{i \rightarrow \infty}\left(a_{n_{i}+1}-a_{n_{i}}\right) \\
& \leq 0 .
\end{aligned}
$$

By (C1), above inequality leads to

$$
\lim _{i \rightarrow \infty}\left\|T_{n_{i}} w_{n_{i}}-T_{n_{i}} z_{n_{i}}\right\|=0 .
$$

Since

$$
\beta_{n_{i}}\left\|z_{n_{i}}-T_{n_{i}} w_{n_{i}}\right\|=\beta_{n_{i}} \gamma_{n_{i}}\left\|g\left(w_{n_{i}}\right)-T_{n_{i}} w_{n_{i}}\right\|,
$$

by $(\mathrm{C} 1)$ and $(\mathrm{C} 2)$ we get

$$
\lim _{i \rightarrow \infty}\left\|z_{n_{i}}-T_{n_{i}} w_{n_{i}}\right\|=0 .
$$

From (3.14) and (3.15), we obtain

$$
\left\|z_{n_{i}}-T_{n_{i}} z_{n_{i}}\right\| \leq\left\|z_{n_{i}}-T_{n_{i}} w_{n_{i}}\right\|+\left\|T_{n_{i}} w_{n_{i}}-T_{n_{i}} z_{n_{i}}\right\| \rightarrow 0
$$

as $i \rightarrow \infty$. We next show that $\lim _{\sup _{i \rightarrow \infty}} b_{n_{i}} \leq 0$. Obviously, it suffices to show that

$$
\limsup _{i \rightarrow \infty}\left\langle g\left(x^{*}\right)-x^{*}, z_{n_{i}}-x^{*}\right\rangle \leq 0 \text {. }
$$

Let $\left\{z_{n_{i_{j}}}\right\}$ be a subsequence of $\left\{z_{n_{i}}\right\}$ such that

$$
\lim _{j \rightarrow \infty}\left\langle g\left(x^{*}\right)-x^{*}, z_{n_{i_{j}}}-x^{*}\right\rangle=\limsup _{i \rightarrow \infty}\left\langle g\left(x^{*}\right)-x^{*}, z_{n_{i}}-x^{*}\right\rangle .
$$

Since $\left\{z_{n_{i_{j}}}\right\}$ is bounded, there exists a subsequence $\left\{z_{n_{i_{j_{p}}}}\right\}$ of $\left\{z_{n_{i_{j}}}\right\}$ and $y \in \mathcal{H}$ such that $z_{n_{i_{p}}} \rightarrow y$. By (3.16), it follows from the condition $(Z)$ of $\left\{T_{n}\right\}$ that $y \in \Gamma$. Here, the equation $x^{*}=P_{\Gamma} g\left(x^{*}\right)$ yields

$$
\limsup _{i \rightarrow \infty}\left\langle g\left(x^{*}\right)-x^{*}, z_{n_{i}}-x^{*}\right\rangle=\lim _{p \rightarrow \infty}\left\langle g\left(x^{*}\right)-x^{*}, z_{n_{i_{j_{p}}}}-x^{*}\right\rangle=\left\langle g\left(x^{*}\right)-x^{*}, y-x^{*}\right\rangle \leq 0 .
$$

By Lemma 2.3, we can conclude that $x_{n} \rightarrow x^{*}$ as $n \rightarrow \infty$. The proof is complete.

Remark 3.1. In Theorem 3.1, if $T_{n}=T$ for all $n \geq 1$, then, by the demiclosedness principle of $T,\left\{T_{n}\right\}$ satisfies the condition (Z).

By above remark, we obtain a strong convergence result for finding a fixed point of a nonexpansive operator $T$.

Corollary 3.1. Let $T: \mathcal{H} \rightarrow \mathcal{H}$ be a nonexpansive operator having a fixed point. Let $g: \mathcal{H} \rightarrow \mathcal{H}$ be a contraction. Suppose that $\left\{x_{n}\right\}$ is a sequence generated iteratively by $x_{0}, x_{1} \in \mathcal{H}$ and

$$
\left\{\begin{array}{l}
w_{n}=x_{n}+\theta_{n}\left(x_{n}-x_{n-1}\right), \\
z_{n}=\left(1-\gamma_{n}\right) T w_{n}+\gamma_{n} g\left(w_{n}\right), \\
x_{n+1}=\left(1-\beta_{n}\right) T w_{n}+\beta_{n} T z_{n}, \quad n \geq 1,
\end{array}\right.
$$


where an inertial parameter $\theta_{n}$ is defined by (3.8) and the real sequences $\left\{\beta_{n}\right\},\left\{\gamma_{n}\right\}$ and $\left\{\tau_{n}\right\}$ satisfy the (C1)-(C3) in Theorem 3.1. Then $\left\{x_{n}\right\}$ converges strongly to $x^{*} \in \operatorname{Fix}(T)$, where $x^{*}=P_{\mathrm{Fix}(T)} g\left(x^{*}\right)$.

Next, we consider the minimization problem in the form of the sum of two convex functions, Problem 1.1. In this situation, we put the following assumptions:

- $f_{1}: \mathbb{R}^{N} \rightarrow \mathbb{R}$ is a convex and differentiable function with a $L$-Lipschitz continuous gradient $\nabla f_{1}$, where $L>0$;

- $f_{2}: \mathbb{R}^{N} \rightarrow \mathbb{R} \cup\{\infty\}$ is a proper lower semi-continuous and convex function;

- $\Omega:=\operatorname{Argmin}\left(f_{1}+f_{2}\right) \neq \emptyset$;

- $g: \mathbb{R}^{N} \rightarrow \mathbb{R}^{N}$ is a contraction, where $0<k<1$

By utilizing Algorithm 1, we obtain the following algorithm for solving Problem 1.1.

Algorithm 2: A Fast Viscosity Forward-Backward Algorithm (FVFBA)

Initialization: Let $\left\{\beta_{n}\right\},\left\{\gamma_{n}\right\},\left\{\tau_{n}\right\},\left\{c_{n}\right\}$ be sequences of positive real numbers and let $\left\{\mu_{n}\right\}$ be a bounded sequence of nonnegative real numbers. Take $x_{0}, x_{1} \in \mathbb{R}^{N}$ arbitrarily.

Iterative steps: For $n \geq 1$, calculate $x_{n+1}$ as follows:

Step 1. Compute an inertial parameter

$$
\theta_{n}= \begin{cases}\min \left\{\mu_{n}, \frac{\tau_{n}}{\left\|x_{n}-x_{n-1}\right\|}\right\} & \text { if } x_{n} \neq x_{n-1} \\ \mu_{n} & \text { otherwise }\end{cases}
$$

Step 2. Compute

$$
\left\{\begin{array}{l}
w_{n}=x_{n}+\theta_{n}\left(x_{n}-x_{n-1}\right), \\
z_{n}=\left(1-\gamma_{n}\right) \operatorname{prox}_{c_{n} f_{2}}\left(I-c_{n} \nabla f_{1}\right) w_{n}+\gamma_{n} g\left(w_{n}\right), \\
x_{n+1}=\left(1-\beta_{n}\right) \operatorname{prox}_{c_{n} f_{2}}\left(I-c_{n} \nabla f_{1}\right) w_{n}+\beta_{n} \operatorname{prox}_{c_{n} f_{2}}\left(I-c_{n} \nabla f_{1}\right) z_{n} .
\end{array}\right.
$$

Update $n:=n+1$ and go to Step 1 .

A convergence result below is definitely a consequence of Theorem 3.1.

Theorem 3.2. A sequence $\left\{x_{n}\right\}$ generated by Algorithm 2 (FVFBA) converges to a point $x^{*} \in \Omega$, where $x^{*}=P_{\Omega} g\left(x^{*}\right)$, provided that the sequences $\left\{\beta_{n}\right\},\left\{\gamma_{n}\right\},\left\{\tau_{n}\right\}$ and $\left\{c_{n}\right\}$ satisfy the following conditions:

(C1) $0<\epsilon_{1} \leq \beta_{n} \leq \epsilon_{2}<1$;

(C2) $0<\gamma_{n}<1$ such that $\lim _{n \rightarrow \infty} \beta_{n} \gamma_{n}=0$ and $\sum_{n=1}^{\infty} \beta_{n} \gamma_{n}=\infty$;

(C3) $\lim _{n \rightarrow \infty} \frac{\tau_{n}}{\beta_{n} \gamma_{n}}=0$;

(C4) $0<c_{n}, c<2 / L$ such that $\lim _{n \rightarrow \infty} c_{n}=c$.

Proof. Let $T_{n}:=\operatorname{prox}_{c_{n} f_{2}}\left(I-c_{n} \nabla f_{1}\right)$ for all $n \geq 1$ and $T:=\operatorname{prox}_{c f_{2}}\left(I-c \nabla f_{1}\right)$. Then, $T_{n}$ and $T$ are nonexpansive operators with $\bigcap_{n=1}^{\infty} \operatorname{Fix}\left(T_{n}\right)=\operatorname{Fix}(T)=\operatorname{Argmin}\left(f_{1}+f_{2}\right)$. Let $\left\{u_{n}\right\}$ be a bounded sequence in $\mathbb{R}^{N}$ such that $\left\|u_{n}-T_{n} u_{n}\right\| \rightarrow 0$ as $n \rightarrow \infty$. If $u$ is a weak cluster point of $\left\{u_{n}\right\}$, then there is a subsequence $\left\{u_{n_{i}}\right\}$ of $\left\{u_{n}\right\}$ such that $u_{n_{i}} \rightarrow u$ as $i \rightarrow \infty$. By Lemma 2.1, we know that $\left\{T_{n}\right\}$ satisfies the NST-condition (I) with $T$. This yields

$$
\lim _{i \rightarrow \infty}\left\|u_{n_{i}}-T u_{n_{i}}\right\|=\lim _{n \rightarrow \infty}\left\|u_{n}-T u_{n}\right\|=0 .
$$

By the demiclosedness of $I-T$ at zero, we get $u \in \operatorname{Fix}(T)=\bigcap_{n=1}^{\infty} \operatorname{Fix}\left(T_{n}\right)$. This implies that $\left\{T_{n}\right\}$ satisfies the condition $(Z)$. Therefore, the result is obtained directly from Theorem 3.1. 


\section{NUMERICAL EXPERIMENTS IN IMAGE RECOVERY}

In this section, we apply the convex minimization problem, Problem 1.1, to the image restoration problem (1.1) via the LASSO model (1.2) by setting

$$
f_{1}(x)=\frac{1}{2}\|y-B x\|_{2}^{2} \text { and } f_{2}(x)=\lambda\|x\|_{1} .
$$

We analyze and illustrate the performance of the FVFBA (Algorithm 2) for the image restoration problem and also compare its efficiency with the FBSA (1.5), the VFBA (1.7) and the FISTA (1.6) by means of Peak Signal-to-Noise Ratio (PSNR) in decibel (dB) [24] and Structural Similarity Index Metric (SSIM) [25]. All experiments and visualizations are done with MATLAB. The maximum iteration number for all methods is fixed at 100 .

Firstly, we test the convergence behavior of the FVFBA by taking the color image (home) with size of $256 \times 256$. Consider Gaussian blur of filter size $9 \times 9$ with standard deviation $\sigma=4$ and noise $10^{-4}$. The values of PSNR (dB) and SSIM for the "home" image corrupted by Gaussian blur are PSNR $=20.532 \mathrm{~dB}$ and SSIM $=0.6803$.

Set the regularization parameter $\lambda=10^{-4}$. The parameters of the FVFBA are chosen as follows: $\beta_{n}=\frac{0.99 n}{n+1}, \gamma_{n}=\frac{1}{50 n}, c_{n}=\frac{n}{n+1}$, and $\tau_{n}=\frac{10^{15}}{n^{2}}$. Let $g(x)=0.95 x$. Now, the expertiments for recovering the "home" image of the FVFBA with different accelerating parameters $\mu_{n}$ are shown in Table 1 and Figures 1 and 2. We also observe from Table 1 and Figure 2 that the parameter $\mu_{n}=\frac{n}{n+1}$ gives the higher values of PSNR and SSIM than the other experiments.

TABLE 1. The values of PSNR and SSIM of restored "home" images by the FVFBA with different accelerating parameters $\mu_{n}$.

\begin{tabular}{ccrr}
\hline Experiments & Accelerating parameters & PSNR & SSIM \\
\hline 1 & $\mu_{n}=0$ & 26.014 & 0.8829 \\
\hline 2 & $\mu_{n}=\frac{t_{n}-1}{t_{n+1}}, t_{1}=1, t_{n+1}=\frac{1+\sqrt{1+4 t_{n}^{2}}}{2}$ & 30.693 & 0.9489 \\
\hline 3 & $\mu_{n}=0.9$ & 29.906 & 0.9412 \\
\hline 4 & $\mu_{n}=\frac{n}{n+2}$ & 31.132 & 0.9528 \\
\hline 5 & $\mu_{n}=\frac{n}{n+1}$ & 31.473 & 0.9546 \\
\hline
\end{tabular}



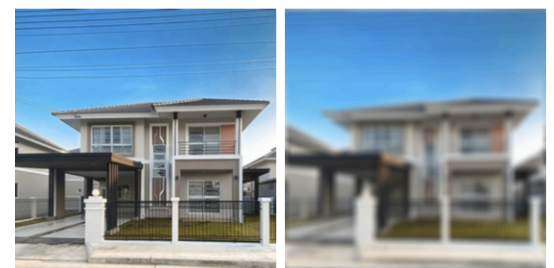

(c) Experiment 1

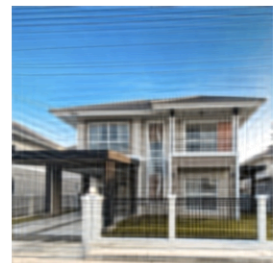

(d) Experiment 2

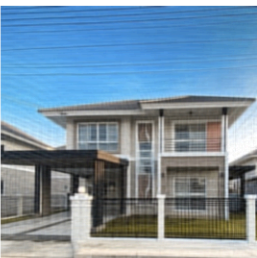

(e) Experiment 3

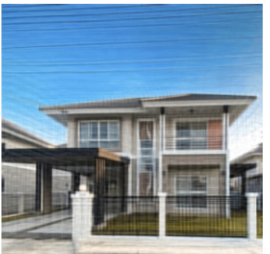

(f) Experiment 4

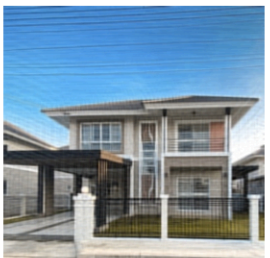

(g) Experiment 5

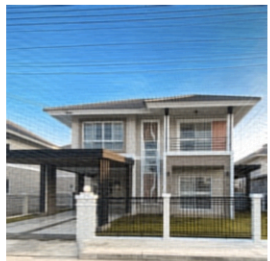

FIGURE 1. Restoration for the "home" image. (a) Original image; (b) Blurry image contaminated by Gaussian blur; (c)-(g) Restored images by the FVFBA with different accelerating parameters.

(a) Peak signal-to-noise ratio (PSNR)

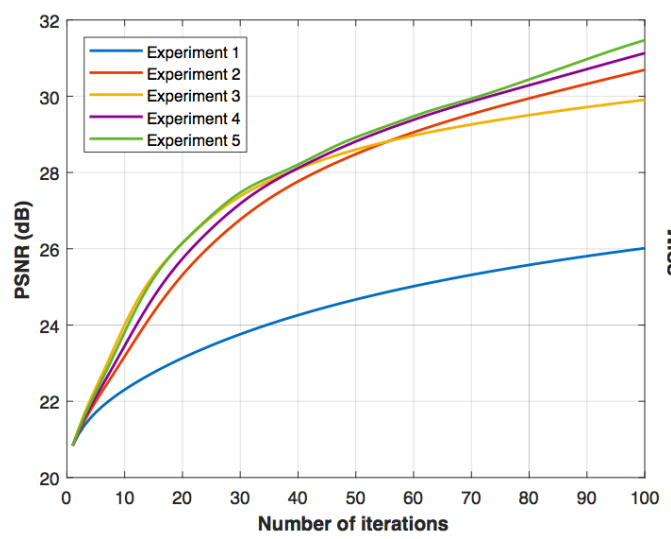

(b) Structural similarity index metric (SSIM)

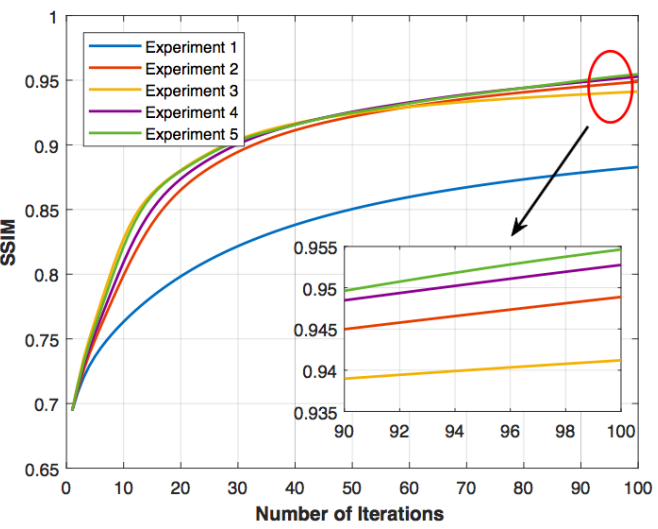

FIGURE 2. Plot of PSNR and SSIM of restored "home" images by the FVFBA.

Next, we compare the performance of the FVFBA (Algorithm 2) with the FBSA (1.5), the VFBA (1.7) and the FISTA (1.6) by means of PSNR and SSIM. We take two color images (bird and butterfly with size of $288 \times 288$ and $256 \times 256$, respectively) from the dataset set 5 [6] and consider two blurring processes with noise $10^{-4}$, i.e., Gaussian blur of filter size $9 \times 9$ with standard deviation $\sigma=10$ and a motion blur specifying with motion length 21 pixels (len $=21)$ and motion orientation $11^{\circ}(\theta=11)$.

Let $\lambda, \beta_{n}, \gamma_{n}, \tau_{n}, c_{n}$ and $g$ be the same as the first test and $\mu_{n}=\frac{n}{n+1}$. Let us see the numerical experiments for recovering the images (bird and butterfly) of the FVFBA (Algorithm 2), the FBSA (1.5), the VFBA (1.7) and the FISTA (1.6) as shown in Figures 3-4. It can be seen that the FVFBA gives the higher values of PSNR and SSIM than the other tested methods. Therefore, the FVFBA has the highest image recovery efficiency comparing with other methods. 
(a) Original Image
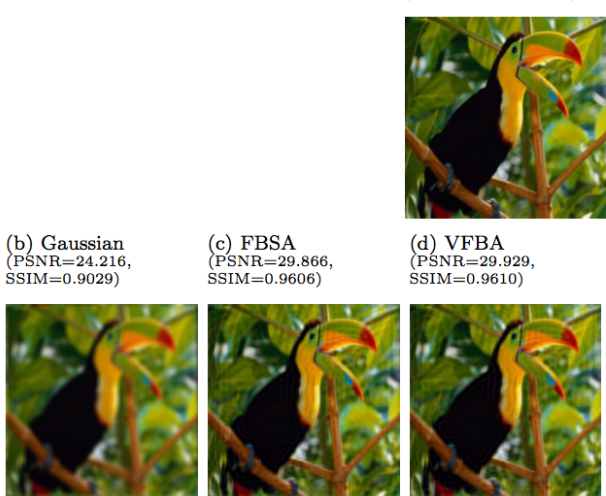

(d) VFBA
(PSNR=29.929,
SSIM $=0.9610)$

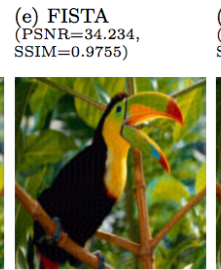

(f) FVFBA

SSIM $=0.9792$ )

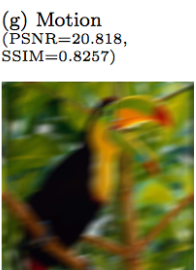

(h) FBSA

(PSNR $=27.499$

$\mathrm{SSIM}=0.9153$ )

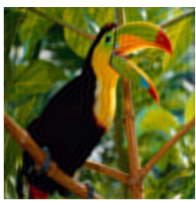

(i) VFBA $(\mathrm{PSNR}=27.585$
$\mathrm{SSIM}=0.9161)$

(j) FISTA $(\mathrm{PSNR}=33.173$,
$\mathrm{SSIM}=0.9561)$
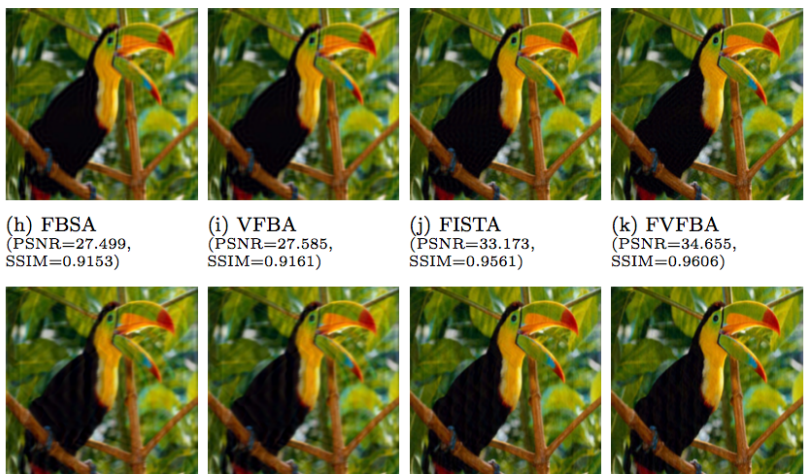

(k) FVFBA PSNR $=34.655$

FIGURE 3. Restoration for the "bird" image. (a) Original image; (b) Blurry image contaminated by Gaussian blur; (c)-(f) Restored images of the image contaminated by Gaussian blur; (g) Blurry image contaminated by Motion blur; $(\mathrm{h})-(\mathrm{k})$ Restored images of the image contaminated by Motion blur.

(a) Original Image
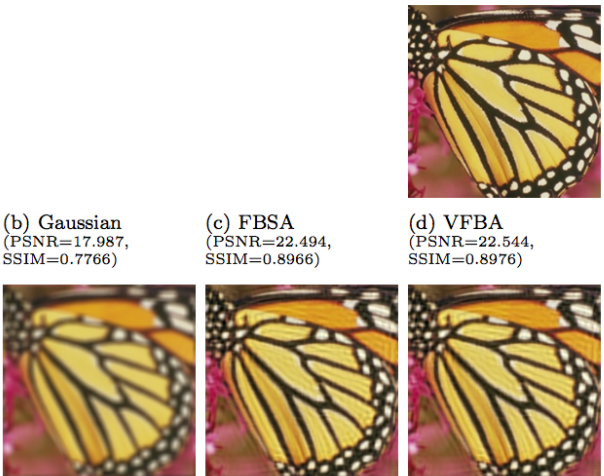

(c) FBSA
(PSNR $=22.494$,

(d) VFBA SSIM $=0.8976$ )

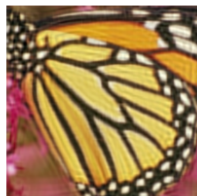

(g) Motion PSNR=15.591 $\operatorname{SSIM}=0.7170$ )

(h) FBSA (PSNR $=21.577$,
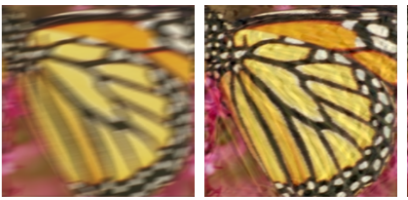

(e) FISTA PIM=0.9456)

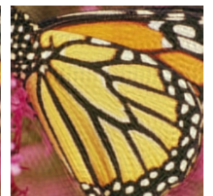

(j) FISTA $\mathrm{PSNR}=27.728$,
$\mathrm{SSIM}=0.9666)$

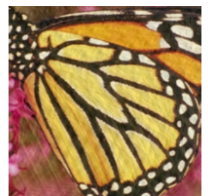

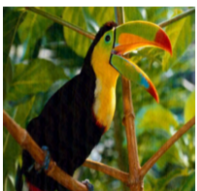

FIGURE 4. Restoration for the "butterfly" image. (a) Original image; (b) Blurry image contaminated by Gaussian blur; (c)-(f) Restored images of the image contaminated by Gaussian blur; (g) Blurry image contaminated by Motion blur; (h)-(k) Restored images of the image contaminated by Motion blur.

\section{CONCLUSION}

In this work, we discuss the convex minimization problem which can be applied in image processing. A relationship between the minimization problem and the fixed point 
problem attracts us to study fixed point methods. We first propose a modified inertial viscosity approximation method for finding a common fixed point of a family of nonexpansive operators in a Hilbert space, and then obtain a strong convergence result under some conditions. After that, we apply the proposed algorithm to solving a convex minimization problem in the form of the sum of two convex functions. As an application, we utilize and analyze our proposed algorithm to the image restoration problem. The numerical experiments show that our algorithm has a higher image recovery efficiency than the forward-backward splitting algorithm, the fast iterative shrinkage-thresholding algorithm and the classical viscosity forward-backward algorithm.

Acknowledgments. This work was supported by Thailand Science Research and Innovation under the project IRN62W0007. P. Jailoka was supported by Post-Doctoral Fellowship of Chiang Mai University, Thailand. We also would like to thank Chiang Mai University for the partial financial support.

\section{REFERENCES}

[1] Aoyama, K.; Kimura, Y. Strong convergence theorems for strongly nonexpansive sequences. Appl. Math. Comput. 217 (2011), 7537-7545

[2] Aoyama, K.; Kohsaka, F.; Takahashi, W. Strong convergence theorems by shrinking and hybrid projection methods for relatively nonexpansive mappings in Banach spaces, In Proceedings of the 5th International Conference on Nonlinear Analysis and Convex Analysis, J. Nonlinear Convex Anal., (2009), 7-26

[3] Bauschke, H. H.; Combettes, P. L. Convex Analysis and Monotone Operator Theory in Hilbert Spaces. Springer, New York, 2011

[4] Beck, A.; Teboulle, M., A fast iterative shrinkage-thresholding algorithm for linear inverse problems. SIAM Journal on Imaging Sciences 2 (2009), 183-202

[5] Bussaban, L.; Suantai, S.; Kaewkhao, A. A parallel inertial S-iteration forward-backward algorithm for regression and classification problems. Carpathian J. Math. 36 (2020), 35-44

[6] Bevilacqua, M.; Roumy, A.; Guillemot, C. and Alberi-Morel, M. L. Low-complexity single-image superresolution based on nonnegative neighbor embedding, In Proceedings British Machine Vision Conference, (2012), http: / /dx.doi.org/10.5244/C.26.135

[7] Combettes, P. L.; Wajs, V. R. Signal recovery by proximal forward-backward splitting. Multiscale Model. Simul. 4 (2005), 1168-1200

[8] Chierchia, G.; Pustelnik, N.; Pesquet, J. C.; Pesquet-Popescu, B. Epigraphical projection and proximal tools for solving constrained convex optimization problems. Part I. Technical report, (2013), http:/ / arxiv.org/abs/1210.5844

[9] Daubechies, I.; Defrise, M.; Mol, C. D. An iterative thresholding algorithm for linear inverse problems with a sparsity constraint. Commun. Pure Appl. Math. 57 (2004), 1413-1457

[10] Figueiredo, M.; Nowak, R. An EM algorithm for wavelet-based image restoration. IEEE Trans. Image Process. 12 (2003), 906-916

[11] Harizanov, S.; Pesquet, J. C.; Steidl, G. Epigraphical projection for solving least squares Anscombe transformed constrained optimization problems. SSVM. (2013), 125-136

[12] Hale, E.; Yin, W.; Zhang, Y. A fixed-point continuation method for $l_{1}$-regularized minimization with applications to compressed sensing. Rice University: Department of Computational and Applied Mathematics, 2007

[13] Lions, P. L.; Mercier, B., Splitting algorithms for the sum of two nonlinear operators. SIAM Journal on Numerical Anal. 16 (1979), no. 6, 964-979

[14] Liang, J.; Schonlieb, C. B. Improving fista: Faster, smarter and greedier, (2018), https://arxiv.org/abs/1811.01430

[15] Moreau, J. J., Fonctions convexes duales et points proximaux dans un espace hilbertien. C. R. Acad. Sci. Paris Sér. A Math. 255 (1962), 2897-2899

[16] Moudafi, A. Viscosity approximation method for fixed-points problems. J. Math. Anal. Appl. 241 (2000), $46-55$

[17] Nesterov, Y. A method for solving the convex programming problem with convergence rate $O\left(1 / k^{2}\right)$. Dokl. Akad. Nauk SSSR 269 (1983), 543-547

[18] Nakajo, K.; Shimoji, K.; Takahashi, W. On strong convergence by the hybrid method for families of mappings in Hilbert spaces. Nonlinear Anal. 71 (2009), No. 1-2, 112-119

[19] Opial, Z. Weak convergence of the sequence of successive approximations for nonexpansive mappings. Bull. Am. Math. Soc. 73 (1967), 591-597 
[20] Polyak, B. T. Some methods of speeding up the convergence of iteration methods. U.S.S.R. Comput. Math. Math. Phys. 4 (1964), 1-17

[21] Saejung, S.; Yotkaew, P. Approximation of zeros of inverse strongly monotone operators in Banach spaces. Nonlinear Anal. 75 (2012), 724-750

[22] Tibshirani, R. Regression shrinkage and selection via the lasso. J. R. Stat. Soc. B Methodol. 58 (1996), 267-288

[23] Takahashi, W., Introduction to Nonlinear and Convex Analysis, Yokohama Publishers, Yokohama, 2009

[24] Thung, K.; Raveendran, P. A survey of image quality measures, In Proceedings of the International Conference for Technical Postgraduates (TECHPOS), Kuala Lumpur, Malaysia, 14-15 December 2009, 1-4

[25] Wang, Z.; Bovik, A. C.; Sheikh, H. R.; Simoncelli, E. P. Image quality assessment: from error visibility to structural similarity. IEEE transactions on image processing 13 (2004), no. 4, 600-612

[26] Xu, H. K., Viscosity approximation methods for nonexpansive mappings. J. Math. Anal. Appl. 298 (2004), 279-291.

DEPARTMENT OF MATHEMATICS

FACULTY OF SCIENCE

CHIANG MAI UNIVERSITY

CHIANG MAI 50200, THAILAND

Email address: pachara.j4@gmail.com

Email address: suthep.s@emu.ac.th

Email address: adisak_h@emu.ac.th 